\title{
Correction to: Predictable events elicit less visual and temporal information uptake in an oddball paradigm
}

\author{
Blake W. Saurels ${ }^{1} \cdot$ Ottmar V. Lipp $^{1,2} \cdot$ Kielan Yarrow $^{3} \cdot$ Derek H. Arnold $^{1}$ \\ Published online: 8 July 2020 \\ (C) The Psychonomic Society, Inc. 2020
}

\section{Correction to: Atten Percept Psychophys https://doi.org/10.3758/s13414-019-01899-x}

The Publisher regrets the following production error. The labels for Figures 2, 4, 5, 6, and 7 describe an older version of the figures. The correct figure labels are as follows:

Fig. 2 Reproduced duration difference scores for the long ( 650 $\mathrm{ms})$ trials. Positive difference scores indicate longer reproduced durations relative to random baseline tests, whereas negative difference scores indicate shorter reproduced durations. Grey bars depict average difference scores. Black error bars depict \pm 1 standard error. Coloured circles depict individual difference scores

Fig. 4 Spatial frequency JND threshold difference scores. Positive difference scores indicate decreased sensitivity relative to random baseline tests, whereas negative difference scores indicate increased sensitivity. Grey bars depict average difference scores. Black error bars depict \pm 1 standard error. Coloured circles depict individual difference scores

Fig. 5 a Reproduced duration difference scores for the long (800 ms) trials. Positive difference scores indicate longer reproduced durations relative to single baseline, whereas negative difference scores indicate shorter reproduced durations. Grey bars depict average difference scores. Black error bars depict \pm 1 standard error. Coloured circles depict individual difference scores. b Difference scores for oddball and repeat tests, relative to random tests (as per Experiment 1). Other details are as for Fig. 5a

Fig. 6 a Spatial frequency JND threshold difference scores. Positive difference scores indicate decreased sensitivity relative to single baseline, whereas negative difference scores indicate increased sensitivity. Grey bars depict average difference scores. Black error bars depict \pm 1 standard error. Coloured circles depict individual difference scores. b Difference scores for oddball and repeat tests, relative to random tests (as per Experiment 2). Other details are as for Fig. 6a

Fig. 7 a Reproduced duration difference scores for each test condition, relative to isolated single tests. Positive scores indicate longer reproduced test durations relative to single baselines, whereas negative difference scores indicate shorter reproduced test durations. Grey bars depict average difference scores. Black error bars depict \pm 1 standard error. Coloured circles depict individual difference scores. b Difference scores for oddball and repeat tests, relative to random tests (as per Experiment 1). Other details are as for Fig. 7a

Publisher's note Springer Nature remains neutral with regard to jurisdictional claims in published maps and institutional affiliations.

The online version of the original article can be found at https://doi.org/ 10.3758/s13414-019-01899-x

Blake W. Saurels

blake.saurels@uq.net.au

1 School of Psychology, The University of Queensland, Saint Lucia, Queensland, Australia

2 School of Psychology, Curtin University, Perth, WA, Australia

3 Department of Psychology, University of London, London, UK 